\title{
A Study on the Second Order Statistics of Nakagami-Hoyt Mobile Fading Channels
}

\author{
Neji Youssef, Member, IEEE, Cheng-Xiang Wang, Student Member, IEEE, and Matthias Pätzold, \\ Senior Member, IEEE
}

\begin{abstract}
In this paper, study of the second order statistics of the Nakagami-Hoyt fading channel model (Nakagami- $q$ model) is considered. Expressions for the level crossing rate (LCR) as well as the average duration of fades (ADF) are derived. It is shown that the obtained analytical quantities best fit the corresponding measurement data for an equivalent mobile satellite channel in the case of an environment with heavy shadowing. This leads to the conclusion that the Nakagami- $q$ model is applicable to realistic mobile communication channels. A simple and efficient deterministic simulation model based on the Rice's sum of sinusoids, which enables the emulation of the fading envelope of the Nakagami- $q$ model with the desired statistics, is also described. A very good agreement is obtained between the simulated, analytical, and experimental statistics.
\end{abstract}

Index Terms-Deterministic channel modeling, fading channels, Nakagami-Hoyt channel, Nakagami- $q$ model, second order statistics.

\section{INTRODUCTION}

I T IS well known that in mobile communications, a profound understanding and accurate modeling of propagation channels are of great importance for both effective system design and related performance analysis. Over the years of wireless communications, and depending on system operating environments, a great number of channel models have been proposed to describe the statistics of the amplitude and phase of multipath fading signals [1]-[3]. The Nakagami- $q$ model is one of the proposed models. This model was introduced by Nakagami [4] as an approximation for the Nakagami- $m$ fading distribution in the range of fading that extends from the one sided Gaussian model to the Rayleigh model. The Nakagami- $q$ distribution is the distribution of the modulus of a complex Gaussian random variable whose components are uncorrelated with zero mean and unequal variances. We should add that this distribution was investigated independently by Hoyt [5], and is sometimes referred to as the Nakagami-Hoyt distribution [6].

Recently, the model is being used more frequently in performance analysis and other studies related to mobile radio communications. For example, Simon et al. [2] considered the Nakagami- $q$ model in a study on error rate performance evaluation of digital communication over generalized fading channels.

Manuscript received May 28, 2002; revised March 30, 2004. The review of this paper was coordinated by Dr. K. Dandekar.

N. Youssef is with the Ecole Superieure des Communications de Tunis, Ariana, Tunisia (e-mail: neji.youssef@ supcom.rnu.tn).

C.-X. Wang and M. Pätzold are with Faculty of Engineering and Science, Agder University College, Grimstad, Norway (e-mail: cheng.wang@hia.no; matthias.paetzold@hia.no).

Digital Object Identifier 10.1109/TVT.2005.851353
Similarly, Annamalai et al. [7] used the model in outage analysis in cellular mobile radio systems. Also, in [8], the model has been combined with the Rice process for the modeling of a two state mobile satellite propagation channel. More recently, the crossing statistics of phase processes and random FM noise of the Nakagami- $q$ channel have been studied [9]. To the best of the authors' knowledge, however, analytical expressions for the second order statistics; i.e., the level-crossing rate (LCR) and the average duration of fades (ADF) in the case of the Nakagami- $q$ model, as well as corresponding experimental verification, have not yet been investigated.

In the present paper, closed form expressions for the second order statistics of the Nakagami- $q$ process are derived. The derived quantities are useful, together with those corresponding to the Rice process [10], for the study of the second order statistics of the combination fading model proposed in [8]. Furthermore, an efficient deterministic simulation model with which the Nakagami- $q$ model can be implemented on a computer is presented. Finally, a comparison of the obtained analytical quantities against measurement data demonstrates the ability of the Nakagami- $q$ model to describe the statistics of real world mobile radio channels.

The paper is organized as follows. In Section II, we briefly review the first order statistics of the envelope and phase processes of the Nakagami- $q$ fading channel model. In Section III, we derive the second order statistics (LCR and ADF) of the model. Section IV deals with the deterministic simulation of the Nakagami- $q$ model. A comparison of the derived statistical quantities with both real-world measurement data and simulated data is discussed in Section V. Finally, in Section VI, we come to the conclusion of the paper.

\section{REVIEW OF THE FIRST ORDER STATISTICS OF THE NAKAGAMI- $q$ FADING MODEL}

In this section, we present a review of the first order statistics of the Nakagami- $q$ fading process which have been investigated in [5]. The instantaneous amplitude, $R(t)$, of this fading process is obtained as the modulus of a complex Gaussian process $\mu(t)=\mu_{1}(t)+j \mu_{2}(t)$. That is,

$$
R(t)=\left|\mu_{1}(t)+j \mu_{2}(t)\right|=\sqrt{\mu_{1}^{2}(t)+\mu_{2}^{2}(t)}
$$

where $\mu_{1}(t)$ and $\mu_{2}(t)$ are uncorrelated zero mean low pass Gaussian processes of variances $\sigma_{1}^{2}$ and $\sigma_{2}^{2}$, respectively. The autocorrelation functions of $\mu_{i}(t)(i=1,2)$ will be denoted by $r_{\mu_{i} \mu_{i}}(\tau)$. In the Nakagami- $q$ model, the autocorrelation functions $r_{\mu_{1} \mu_{1}}(\tau)$ and $r_{\mu_{2} \mu_{2}}(\tau)$ can have different shapes. Hence, 
since $\sigma_{i}^{2}=r_{\mu_{i} \mu_{i}}(0)$, it follows that $\sigma_{1}^{2}$ and $\sigma_{2}^{2}$ can be different. The probability density function (PDF) of the Nakagami- $q$ process $R(t), p_{R}(z)$, is given by [5]

$$
\begin{aligned}
p_{R}(z)=\frac{z}{\sigma_{1} \sigma_{2}} \exp ( & \left.-\frac{z^{2}}{4}\left(\frac{1}{\sigma_{1}^{2}}+\frac{1}{\sigma_{2}^{2}}\right)\right) \\
& \times I_{0}\left(\frac{z^{2}}{4}\left(\frac{1}{\sigma_{2}^{2}}-\frac{1}{\sigma_{1}^{2}}\right)\right), \quad z \geq 0
\end{aligned}
$$

where $I_{0}(\cdot)$ designates the zeroth-order modified Bessel function of the first kind. Note that under the restriction that $\sigma_{1}=\sigma_{2}$, the PDF of the process $R(t)$ reduces to the Rayleigh distribution [11]. Also, the PDF (2) contains the one-sided Gaussian density as a special case [5]. This can be shown by writing the $\operatorname{PDF} p_{R}(z)$ in the following form [5]

$$
\begin{aligned}
p_{R}(z)=\frac{2}{\sqrt{2 \pi\left(\sigma_{1}^{2}-\sigma_{2}^{2}\right)}} \\
\quad \times \exp \left(-\frac{z^{2}}{2 \sigma_{1}^{2}}\right) \sqrt{2 \pi X} \exp (-X) I_{0}(X)
\end{aligned}
$$

where $X=\left(z^{2} / 4\right)\left(\left(1 / \sigma_{2}^{2}\right)-\left(1 / \sigma_{1}^{2}\right)\right)$. Then, in the limit $\sigma_{2}^{2} \rightarrow$ $0\left(\sigma_{1}^{2} \neq 0\right)$; i.e., $X \rightarrow \infty$, we have $\sqrt{2 \pi X} \exp (-X) I_{0}(X) \rightarrow$ 1 , and thus the PDF (3) leads to the one-sided Gaussian density given by $p_{R}(z)=\left(2 /\left(\sqrt{2 \pi} \sigma_{1}\right)\right) \exp \left(-\left(z^{2}\right) /\left(2 \sigma_{1}^{2}\right)\right), z \geq 0$. On the other hand, the Nakagami- $q$ model allows also the approximation of the Nakagami- $m$ fading channel in the range of the parameter $m$, given by $0.5 \leq m \leq 1$, by the following choice of parameters [4]

$$
\Omega=\frac{\sigma_{1}^{2}+\sigma_{2}^{2}}{2}
$$

and

$$
m=\frac{\left(\sigma_{1}^{2}+\sigma_{2}^{2}\right)^{2}}{\left(\sigma_{1}^{2}+\sigma_{2}^{2}\right)^{2}+\left(\sigma_{1}^{2}-\sigma_{2}^{2}\right)^{2}}
$$

where $\Omega=E\left(R^{2}\right)$ and $m=\left\{E\left(R^{2}\right)\right\}^{2} / \operatorname{Var}\left(R^{2}\right)$ are the two parameters describing the Nakagami- $m$ process $R(t)$, the PDF of which is given in [4]. Here, $E(\cdot)$ denotes statistical average, while $\operatorname{Var}(\cdot)$ stands for the variance. The influence of $\rho=$ $\sigma_{2}^{2} / \sigma_{1}^{2}$ on the PDF $p_{R}(z)$ is shown in Fig. 1.

Concerning the phase process, $\vartheta(t)=\arctan \left(\mu_{2}(t) / \mu_{1}(t)\right)$, encountered in the Nakagami- $q$ fading model, its PDF is given by [5]

$$
p_{\vartheta}(\theta)=\frac{\sigma_{1} \sigma_{2}}{2 \pi\left(\sigma_{2}^{2} \cos ^{2}(\theta)+\sigma_{1}^{2} \sin ^{2}(\theta)\right)}, \quad-\pi \leq \theta<\pi .
$$

Note that the case $\sigma_{1}^{2}=\sigma_{2}^{2}$ yields a uniformly distributed phase process in the interval $[-\pi, \pi)$. In the limit $\sigma_{1}^{2} \rightarrow 0, p_{\vartheta}(\theta)$ becomes

$$
p_{\vartheta}(\theta)=\frac{1}{2}(\delta(\theta-\pi / 2)+\delta(\theta+\pi / 2))
$$

while the case $\sigma_{2}^{2} \rightarrow 0$ yields the following PDF:

$$
p_{\vartheta}(\theta)=\frac{1}{2}(\delta(\theta)+\delta(\theta+\pi)) .
$$

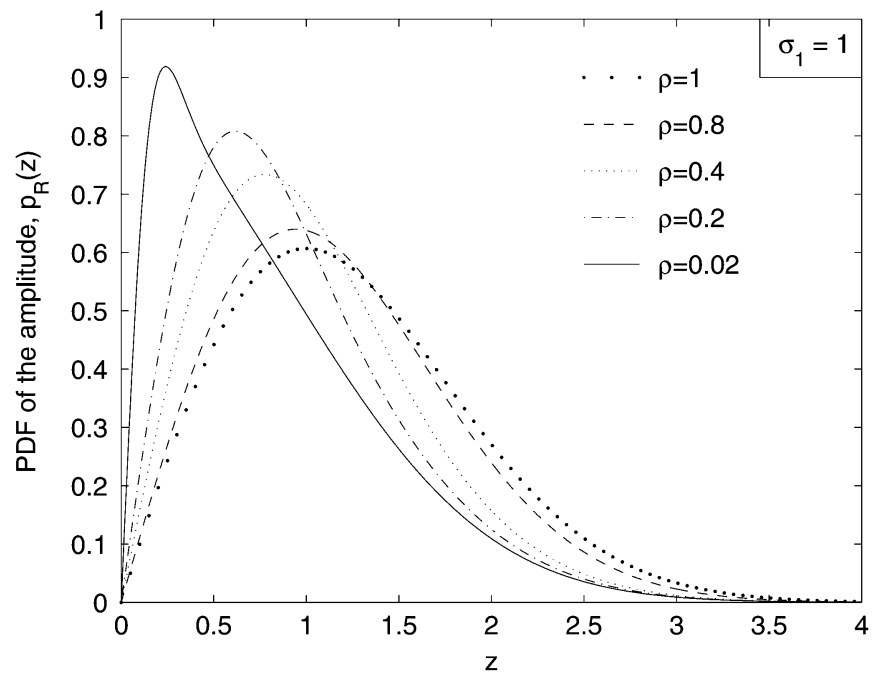

Fig. 1. The $\operatorname{PDF} p_{R}(z)$ as a function of $\rho$.

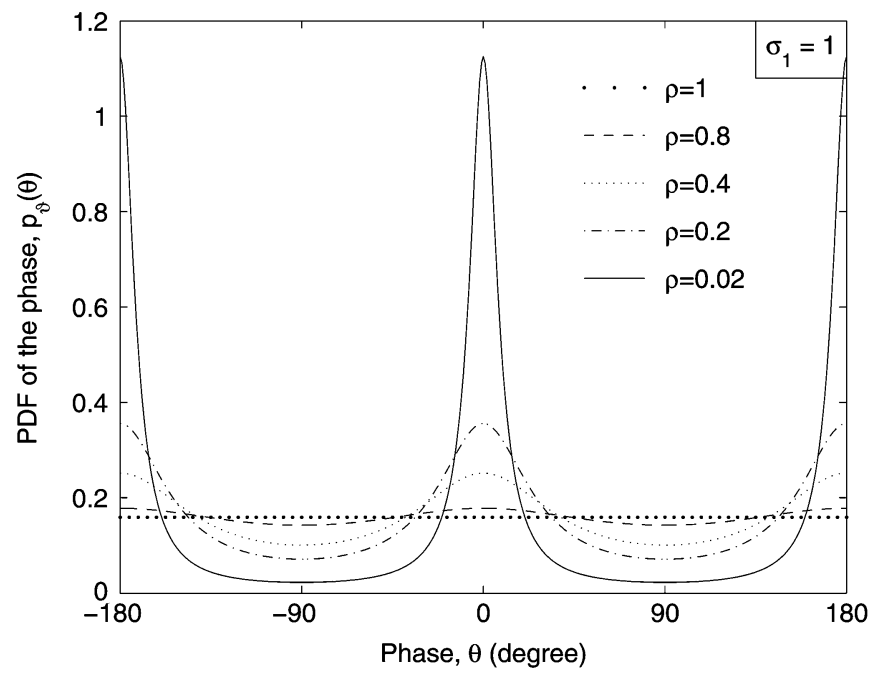

(a)

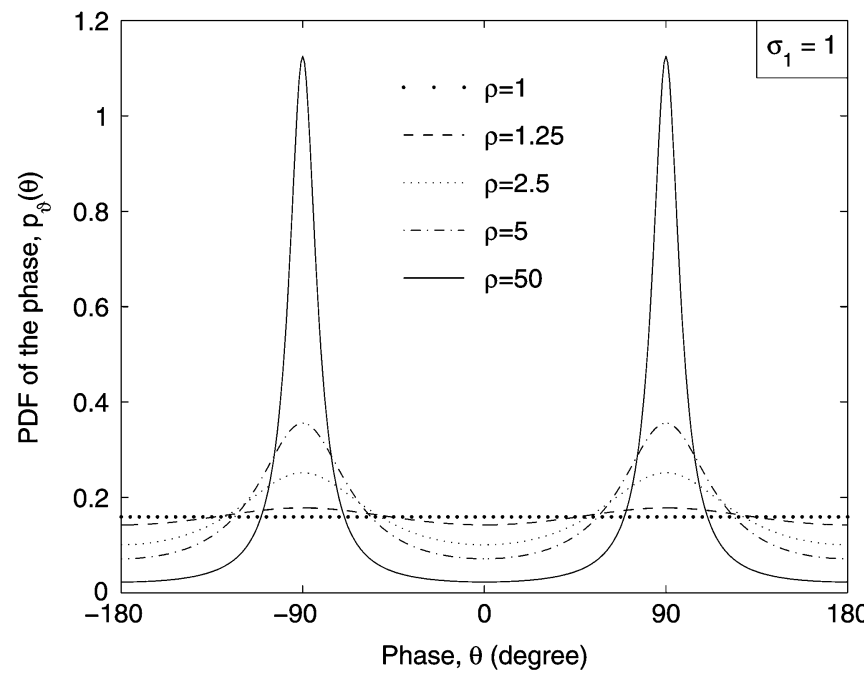

(b)

Fig. 2. The PDF $p_{\vartheta}(\theta)$ as a function of $\rho$ : (a) $\rho \leq 1$ and (b) $\rho \geq 1$. 
The influence of the parameter $\rho=\sigma_{2}^{2} / \sigma_{1}^{2}$ on the phase PDF $p_{\vartheta}(\theta)$ is shown in Fig. 2.

Since the Nakagami- $m$ distribution approximates the Nakagami- $q$ distribution and vice versa, it is worthwhile to present a brief comparison between the two models. It is well known that while the Nakagami- $m$ model offers features of analytical convenience with respect to the Nakagami- $q$ model, it does not address the phase term statistics which is of great importance in coherent communications. Concerning the simulation, the Nakagami-q model can be simulated accurately by using the classical filter method or the concept of deterministic channel modeling [12], since the underlying model is derived directly from uncorrelated Gaussian processes. However, correlated Nakagami- $m$ fading processes cannot, in general, be simulated directly by applying these methods. Therefore, in recent years, several works have addressed the topic of simulating correlated Nakagami- $m$ processes using different approaches [13]-[16]. Here, we mention that a simulation model for the Nakagami- $q$ process can be considered as an approximated simulation model for the Nakagami- $m$ process, the parameters of which are obtained from the relationships given by (4) and (5). In this case, the autocorrelation function of the approximated squared envelope of the Nakagami- $m$ fading channel is the same as that corresponding to the Nakagami- $q$ model; i.e., $r_{R^{2} R^{2}}(\tau)=\sigma_{1}^{4}+\sigma_{2}^{4}+2 \sigma_{1}^{2} \sigma_{2}^{2}+2\left(r_{\mu_{1} \mu_{1}}^{2}(\tau)+r_{\mu_{2} \mu_{2}}^{2}(\tau)\right)$. As was pointed out in [17], so far there is no analytical model, proposed on a physical basis, for the temporal autocorrelation function of the Nakagami- $m$ channel. We add that the importance and usefulness of the equivalence between channel models were also addressed in [3].

\section{SECOND ORDER STATISTICS}

In this section, we consider the derivation of the LCR and the ADF of the Nakagami- $q$ fading process. These quantities are also called second order statistics because they take into consideration the time dimension. In addition to their importance in providing statistical information on the error bursts [18], [19], these quantities are also useful for computing transition probabilities in Markov modeling of fading channels [20], and in velocity estimation of mobile units [21]. Using the traditional PDF-based approach, an analytical expression for the LCR of the process $R(t)$, denoted by $N_{R}(r)$, can be obtained by solving the following integral [10]

$$
N_{R}(r)=\int_{0}^{\infty} \dot{z} p_{R \dot{R}}(r, \dot{z}) d \dot{z}
$$

where $p_{R \dot{R}}(r, \dot{z})$ is the joint PDF (JPDF) of $R(t)$ and its time derivative $\dot{R}(t)$ at the same time and at the level $z=r$. The characteristic function based approach, proposed in [22], can also be used for the derivation of $N_{R}(r)$. For obtaining $N_{R}(r)$ from (9), it is necessary to first calculate an expression for $p_{R \dot{R}}(z, \dot{z})$. This can be done by deriving the JPDF, $p_{R \dot{R} \vartheta \dot{\vartheta}}(z, \dot{z}, \theta, \dot{\theta})$, of the processes $R(t), \dot{R}(t), \vartheta(t)$ and $\dot{\vartheta}(t)$, based on the multivariate Gaussian distribution, $p_{\mu_{1} \mu_{2} \dot{\mu}_{1} \dot{\mu}_{2}}\left(x_{1}\right.$, $\left.x_{2}, \dot{x}_{1}, \dot{x}_{2}\right)$ of the processes $\mu_{1}(t), \dot{\mu}_{1}(t), \mu_{2}(t), \dot{\mu}_{2}(t)$, and the transformation of the Cartesian coordinates $\left(x_{1}, x_{2}\right)$ to polar coordinates $(z, \theta)$. This results in $p_{R \dot{R} \vartheta \dot{\vartheta}}(z, \dot{z}, \theta, \dot{\theta})=|J|^{-1}$ $p_{\mu_{1} \mu_{2} \dot{\mu}_{1} \dot{\mu}_{2}}(z \cos (\theta), z \sin (\theta), \dot{z} \cos (\theta)-z \dot{\theta} \sin (\theta), \dot{z} \sin (\theta)+$ $z \dot{\theta} \cos (\theta)$ ), where $J=-1 / z^{2}$ is the Jacobian of the transformation. For a symmetrical Doppler power spectral density, where $\mu_{i}(t)$ and $\dot{\mu}_{i}(t) \quad(i=1,2)$ are in pairs uncorrelated, it can be shown that $p_{\mu_{1} \mu_{2} \dot{\mu}_{1} \dot{\mu}_{2}}\left(x_{1}, x_{2}, \dot{x}_{1}, \dot{x}_{2}\right)=$ $\left(4 \pi^{2} \sigma_{1} \sigma_{2} \sqrt{\beta_{1} \beta_{2}}\right)^{-1} \exp \left(-1 / 2\left(x_{1}^{2} / \sigma_{1}^{2}+x_{2}^{2} / \sigma_{2}^{2}+\dot{x}_{1}^{2} / \beta_{1}+\right.\right.$ $\left.\dot{x}_{2}^{2} / \beta_{2}\right)$ ), where $\beta_{i}$ is the variance of the process $\dot{\mu}_{i}(t)$ $(i=1,2)$. The application of that transformation then results in the following expression for the JPDF $p_{R \dot{R} \vartheta \dot{\vartheta}}(z, \dot{z}, \theta, \dot{\theta})$

$$
\begin{aligned}
& p_{R \dot{R} \vartheta \dot{\vartheta}}(z, \dot{z}, \theta, \dot{\theta}) \\
&=\frac{z^{2}}{4 \pi^{2} \sigma_{1} \sigma_{2} \sqrt{\beta_{1} \beta_{2}}} \exp \left(-\left[\frac{z^{2} \cos ^{2}(\theta)}{2 \sigma_{1}^{2}}\right.\right. \\
& \quad+\frac{z^{2} \sin ^{2}(\theta)}{2 \sigma_{2}^{2}}+\frac{\dot{z}^{2} \cos ^{2}(\theta)}{2 \beta_{1}} \\
& \quad+\frac{\dot{z}^{2} \sin ^{2}(\theta)}{2 \beta_{2}}+\left(\frac{z^{2} \cos ^{2}(\theta)}{2 \beta_{2}}+\frac{z^{2} \sin ^{2}(\theta)}{2 \beta_{1}}\right) \dot{\theta}^{2} \\
&\left.\left.+z \dot{z} \sin (\theta) \cos (\theta)\left(\frac{1}{\beta_{2}}-\frac{1}{\beta_{1}}\right) \dot{\theta}\right]\right)
\end{aligned}
$$

where $0 \leq z<\infty,-\infty<\dot{z}<\infty,-\pi \leq \theta<\pi$, and $-\infty<$ $\dot{\theta}<\infty$. The quantity $\beta_{i}(i=1,2)$ can be expressed as $\beta_{i}=$ $-\ddot{r}_{\mu_{i} \mu_{i}}(0)$, i.e., $\beta_{i}$ represents the negative curvature of the autocorrelation function $r_{\mu_{i} \mu_{i}}(\tau)$ at $\tau=0$. The JPDF $p_{R \dot{R}}(z, \dot{z})$ of the processes $R(t)$ and $\dot{R}(t)$ is then computed as

$$
\begin{aligned}
p_{R \dot{R}}(z, \dot{z}) & \int_{-\infty}^{\infty} \int_{0}^{2 \pi} p_{R \dot{R} \vartheta \dot{\vartheta}}(z, \dot{z}, \theta, \dot{\theta}) d \theta d \dot{\theta} \\
= & \frac{z}{(2 \pi)^{3 / 2} \sigma_{1} \sigma_{2}} \\
& \times \int_{0}^{2 \pi} \exp \left[-\frac{z^{2}}{2 \sigma_{1}^{2} \sigma_{2}^{2}}\left(\sigma_{2}^{2} \cos ^{2}(\theta)+\sigma_{1}^{2} \sin ^{2}(\theta)\right)\right] \\
& \times \exp \left[-\frac{\dot{z}^{2}}{2\left(\beta_{2}+\left(\beta_{1}-\beta_{2}\right) \cos ^{2}(\theta)\right)}\right] \\
& \times \frac{1}{\sqrt{\left(\beta_{2}+\left(\beta_{1}-\beta_{2}\right) \cos ^{2}(\theta)\right)}} d \theta .
\end{aligned}
$$

Note that $p_{R \dot{R}}(z, \dot{z}) \neq p_{R}(z) \cdot p_{\dot{R}}(\dot{z})$, leading to the conclusion that $R(t)$ and $\dot{R}(t)$ are not statistically independent. This result is different from that obtained for the corresponding cases of Rayleigh [11], Rice [10], and Nakagami- $m$ [23] fading models, where $R(t)$ and $\dot{R}(t)$ have been shown to be statistically independent. Moreover, for these three models, $\dot{R}(t)$ has been shown to be Gaussian distributed. For the Nakagami- $q$ model, the PDF of $\dot{R}(t)$ is obtained from (11) as

$$
\begin{aligned}
p_{\dot{R}}(\dot{z}) & =\int_{0}^{2 \pi} \frac{2}{\sqrt{2 \pi\left(\beta_{2}+\left(\beta_{1}-\beta_{2}\right) \cos ^{2}(\theta)\right)}} \\
& \times \exp \left(-\frac{\dot{z}^{2}}{2\left(\beta_{2}+\left(\beta_{1}-\beta_{2}\right) \cos ^{2}(\theta)\right)}\right) p_{\vartheta}(\theta) d \theta
\end{aligned}
$$

where $p_{\vartheta}(\theta)$ is given by (6). 


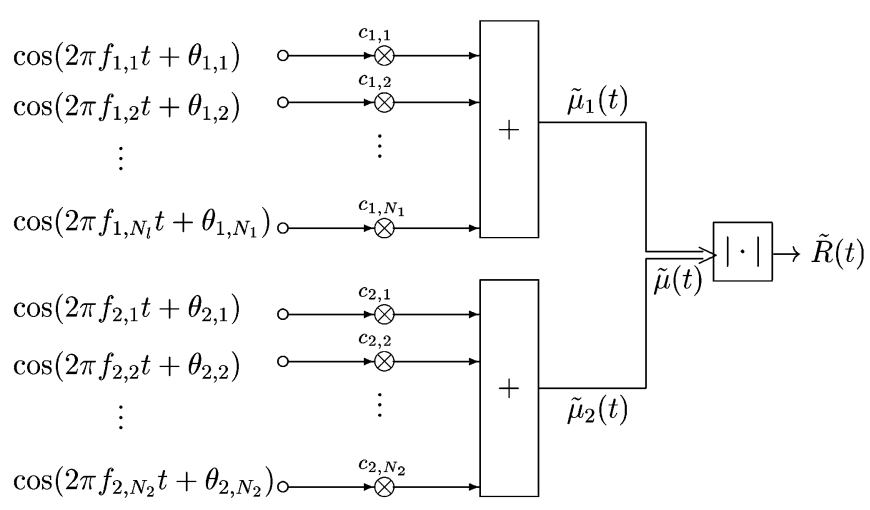

Fig. 3. Structure of the deterministic simulation system.

Now, the LCR $N_{R}(r)$ of the Nakagami- $q$ process follows immediately from (9), and can be expressed by

$$
\begin{aligned}
N_{R}(r)= & \frac{r}{(2 \pi)^{3 / 2} \sigma_{1} \sigma_{2}} \\
& \times \int_{0}^{2 \pi} \exp \left[-\frac{r^{2}}{2 \sigma_{1}^{2} \sigma_{2}^{2}}\left(\sigma_{2}^{2} \cos ^{2}(\theta)+\sigma_{1}^{2} \sin ^{2}(\theta)\right)\right] \\
& \times \sqrt{\left(\beta_{2}+\left(\beta_{1}-\beta_{2}\right) \cos ^{2}(\theta)\right)} d \theta .
\end{aligned}
$$

In case of $\sigma_{1}^{2}=\sigma_{2}^{2}=\sigma^{2}\left(\beta_{1}=\beta_{2}=\beta\right)$, it is easy to show that (13) reduces to the well known expression of the LCR corresponding to the Rayleigh fading channel ( [11], (1.3.35)).

Another statistical quantity that helps to quantify the characteristics of correlated fading channels is the ADF. The ADF $T_{R-}(r)$ is the expected value for the length of time intervals over which the signal envelope $R(t)$ is below a specified level $r$. In general, the ADF $T_{R-}(r)$ is defined by [11]

$$
T_{R-}(r)=\frac{P_{R-}(r)}{N_{R}(r)}
$$

where $P_{R-}(r)$ indicates the probability that the process $R(t)$ is found below the level $r$. An expression for $P_{R_{-}}(r)$ can be derived from (2) according to

$$
\begin{aligned}
P_{R-}(r)= & \int_{0}^{r} p_{R}(z) d z \\
= & \int_{0}^{r} \frac{z}{\sigma_{1} \sigma_{2}} \exp \left(-\frac{z^{2}}{4}\left(\frac{1}{\sigma_{1}^{2}}+\frac{1}{\sigma_{2}^{2}}\right)\right) \\
& \times I_{0}\left(\frac{z^{2}}{4}\left(\frac{1}{\sigma_{2}^{2}}-\frac{1}{\sigma_{1}^{2}}\right)\right) d z .
\end{aligned}
$$

By substituting (13) and (15) into (14), the ADF $T_{R_{-}}(r)$ of the Nakagami- $q$ process $R(t)$ can be evaluated.

\section{SimUlation SYSTEM}

We propose, in this paper, to simulate the Nakagami- $q$ process by using the concept of deterministic channel modeling based on Rice's sum of sinusoids. According to that principle, the simulation system of the Nakagami- $q$ process has the structure shown in Fig. 3, where the Gaussian processes $\mu_{i}(t)(i=1,2)$ are approximated by

$$
\tilde{\mu}_{i}(t)=\sum_{n=1}^{N_{i}} c_{i, n} \cos \left(2 \pi f_{i, n} t+\theta_{i, n}\right) .
$$

The quantities $c_{i, n}, f_{i, n}$, and $\theta_{i, n}$ in (16) are called the gains, discrete Doppler frequencies, and phases, respectively, and $N_{i}$ denotes the number of sinusoids used for the generation of $\tilde{\mu}_{i}(t)$. All these parameters have to be determined such that the process given by (16) has the desired statistics. For the computation of these quantities, several methods have been proposed in [12]. Here, we use the so-called Method of Exact Doppler Spread (MEDS). The MEDS is discussed in [12], and its use is restricted to the Jakes Doppler power spectral density [11]. In this case, the corresponding autocorrelation function of the Gaussian processes $\mu_{i}(t)(i=1,2)$ is given by

$$
r_{\mu_{i} \mu_{i}}(\tau)=\sigma_{i}^{2} J_{0}\left(2 \pi f_{\max _{i}} \tau\right)
$$

where $J_{0}(\cdot)$ is the zeroth-order Bessel function of the first kind and $f_{\max _{i}}$ denotes the maximum Doppler frequency of the process $\mu_{i}(t)$. The quantity $\beta_{i}(i=1,2)$ can be obtained from (17) as $\beta_{i}=-\ddot{r}_{\mu_{i} \mu_{i}}(0)=2\left(\pi \sigma_{i} f_{\max _{i}}\right)^{2}$. According to the MEDS [12], the corresponding formulas for the determination of $c_{i, n}$ and $f_{i, n}$ are given by $c_{i, n}=\sigma_{i} \sqrt{2 / N_{i}}$ and $f_{i, n}=$ $f_{\max _{i}} \sin \left[\left(\pi /\left(2 N_{i}\right)\right)(n-(1 / 2))\right]$ respectively, while the phases $\theta_{i, n}$ are realizations of a random variable that is uniformly distributed in the interval $[0,2 \pi)$. Here, we should add that the values of the maximum Doppler frequencies, $f_{\text {max }_{i}}(i=1,2)$, can be obtained according to the relation

$$
f_{\max _{2}}=\frac{\sigma_{1}}{\sigma_{2}} \sqrt{\frac{\beta_{2}}{\beta_{1}}} f_{\max }
$$

where $f_{\max }=f_{\max _{1}}$. This simulation procedure which allows, as will be shown in the following, to simulate the Nakagami$q$ process with high precision, can also be useful for the approximation of correlated Nakagami- $m$ fading envelopes, $0.5 \leq m \leq 1$. The parameters characterizing the corresponding simulated Nakagami- $m$ channel are given by (4) and (5). In comparison with the method proposed in [13] for the simulation of Nakagami- $m$ processes, $0.5 \leq m \leq 1$, we can say that the present method is much simpler in terms of computational complexity, and the simulated autocorrelation function offers a better match to the exact analytical one. However, concerning the PDF of the Nakagami- $m$ model, we have verified that the approximation is quite good only for large values of envelope levels, and the method proposed in [13] yields a better approximation of the Nakagami- $m$ PDF. For completeness, we mention that the filter method can also be used for the simulation of Nakagami- $q$ processes.

\section{Adaptation to Measurement Data}

To demonstrate the validation of the Nakagami- $q$ channel model for describing the statistics of real-world mobile fading channels, an adaptation of the complementary cumulative distribution function (CDF), LCR, and ADF to measurement results is performed. The measurement results of the complementary 
TABLE I

OPTIMIZED PARAMETERS OF THE ANALYTICAL NAKAGAMI- $Q$ MODEL FOR RURAL AREAS WITH HEAVY SHADOWING

\begin{tabular}{l|l|l|l|l}
\hline Parameters & $\sigma_{1}^{2}$ & $\sigma_{2}^{2}$ & $\beta_{1}$ & $\beta_{2}$ \\
\hline Optimized values & 0.10391 & 0.030488 & 1103.4298 & 1091.5206 \\
\hline
\end{tabular}

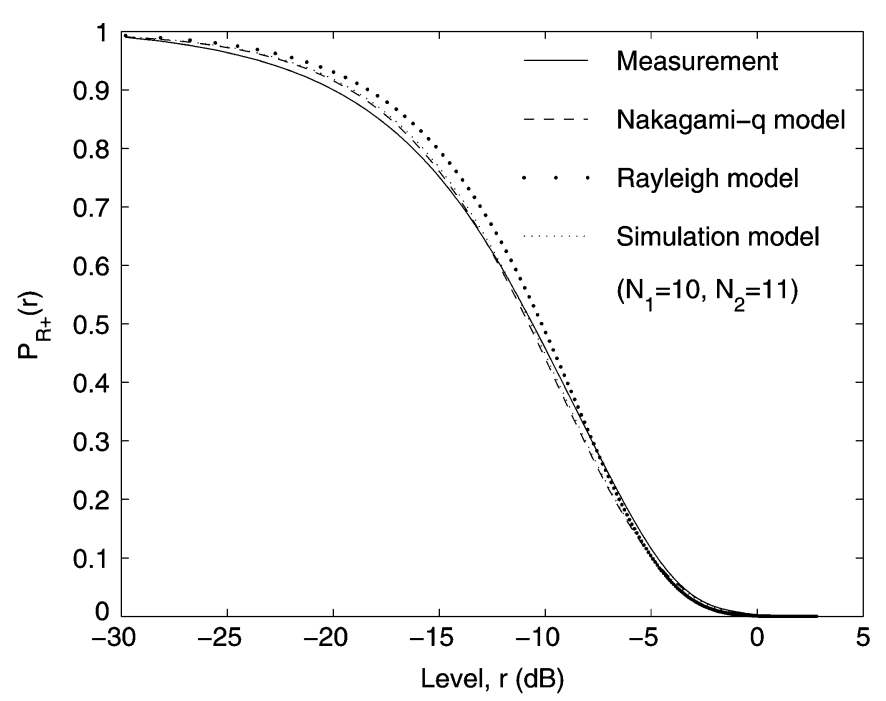

Fig. 4. Complementary CDF $P_{R_{+}}(r)$ for heavy shadowing.

CDF $P_{R_{+}}^{*}(r)$, LCR $N_{R}^{*}(r)$, and $\operatorname{ADF} T_{R_{-}}^{*}(r)$ considered as object functions for the optimization of the parameters of the Nakagami- $q$ channel model are adopted from [24]. An appropriate error norm for our purpose which provides us with a measure of the difference between the analytical quantities and the measured ones is given by

$$
\begin{aligned}
& E_{2}(\Psi):=\left(\sum_{m=1}^{M}\left\{W_{1}\left(r_{m}\right) \cdot\left[P_{R+}\left(r_{m}\right)-P_{R+}^{*}\left(r_{m}\right)\right]\right\}^{2}\right)^{1 / 2} \\
& +\frac{1}{f_{\max }}\left(\sum_{m=1}^{M}\left\{W_{2}\left(r_{m}\right) \cdot\left[N_{R}\left(r_{m}\right)-N_{R}^{*}\left(r_{m}\right)\right]\right\}^{2}\right)^{1 / 2}
\end{aligned}
$$

where $\Psi$ denotes the parameter vector $\Psi=\left(\sigma_{1}, \sigma_{2}, \beta_{1}, \beta_{2}\right), M$ is the number of measurement values, and $W_{1}(\cdot)$ and $W_{2}(\cdot)$ are appropriate weighting functions which are defined here, for simplicity, by scaled versions of the reciprocals of $P_{R+}^{*}(\cdot)$ and $N_{R}^{*}(\cdot)$, respectively. Observe that we only consider $N_{R}\left(r_{m}\right)$ and $P_{R+}\left(r_{m}\right)=1-P_{R-}\left(r_{m}\right)$ in the above error function, and we ignore $T_{R-}\left(r_{m}\right)$ because $T_{R-}\left(r_{m}\right)$ is completely defined by $P_{R_{-}}\left(r_{m}\right)$ and $N_{R}\left(r_{m}\right)$ - see (14). The minimization of the error norm $E_{2}(\Psi)$ can be performed by applying any elaborate numerical optimization procedure. Here, we have used the so-called quasi-Newton algorithm [25], [26]. The result of the minimization of (19) is shown in Table I, where the optimized parameters of the Nakagami- $q$ model are listed for the equivalent mobile satellite channel. Following [3], the Nakagami- $m$ channel parameters corresponding to the set of measurement data employed in this paper can directly be estimated from the optimized parameters of the Nakagami- $q$ channel using (4) and

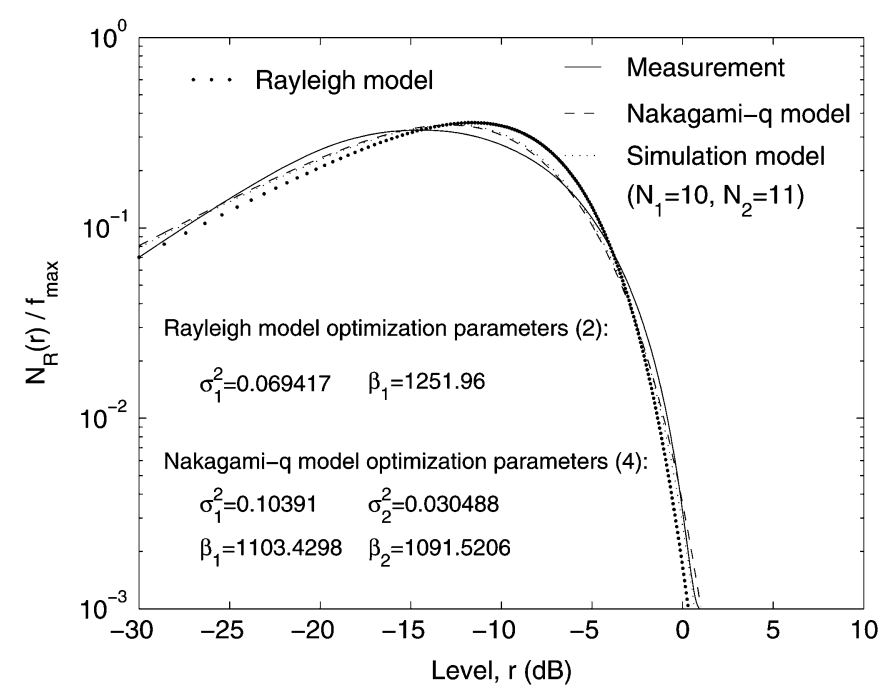

Fig. 5. Normalized level-crossing rate $N_{R}(r) / f_{\max }$ for heavy shadowing.

(5). This results in the values $m=0.77$ and $\Omega=0.067$. It is interesting to note that the value of $m$ is approximately the same as that obtained for the model proposed in [3, Table III] for the heavy shadowing environment.

The results of the fitted, simulated, and measured complementary CDF are compared in Fig. 4. The simulation results shown are obtained by using the optimized parameters given in Table I, and the values of $N_{1}$ and $N_{2}$ are selected to be 10 and 11 , respectively. We see that the fitted complementary CDF $P_{R+}(r)$ is in remarkable good agreement with the underlying measured and simulated results. For further comparison, the fitted complementary CDF of the Rayleigh model is also plotted; thus we can observe that the Nakagami- $q$ model is in a better coincidence with measurement data than the Rayleigh model. Similarly, the normalized measured LCR $N_{R}^{*}(r) / f_{\max }$ and the normalized measured ADF $T_{R}^{*}(r) \cdot f_{\max }$ are shown in Figs. 5 and 6, respectively. In these figures, the resulting normalized LCR and ADF of the analytical Nakagami- $q$ model and the simulation model are also plotted for the purpose of comparison. All the integrals involved in the analytical expressions of the LCR and ADF are computed by using the trapezoidal method [27]. The corresponding values of the maximum Doppler frequencies, deduced from the parameters given in Table I, are $f_{\max }=f_{\max _{1}}=23.19 \mathrm{~Hz}$, and $f_{\max _{2}}=42.58 \mathrm{~Hz}$. Although the assumption that the Gaussian processes $\mu_{1}(t)$ and $\mu_{2}(t)$ may have different maximum Doppler frequencies lacks a clear physical basis, it increases the flexibility of the model and enables better fitting of measurement data. The results show that the analytical Nakagami- $q$ model and the simulation results are in good agreement with the measurement data. In particular, it is interesting to observe from Fig. 6, that the analytical ADF of the Nakagami- $q$ model fits clearly better the measured ADF than does the ADF of the Rayleigh channel, especially when the threshold $r$ is between $-5 \mathrm{~dB}$ and $0 \mathrm{~dB}$. This allows us to conclude that the advantage of the Nakagami- $q$ model over the Rayleigh model, for this realistic mobile satellite channel, appears mainly in terms of the ADF. By comparison with the 


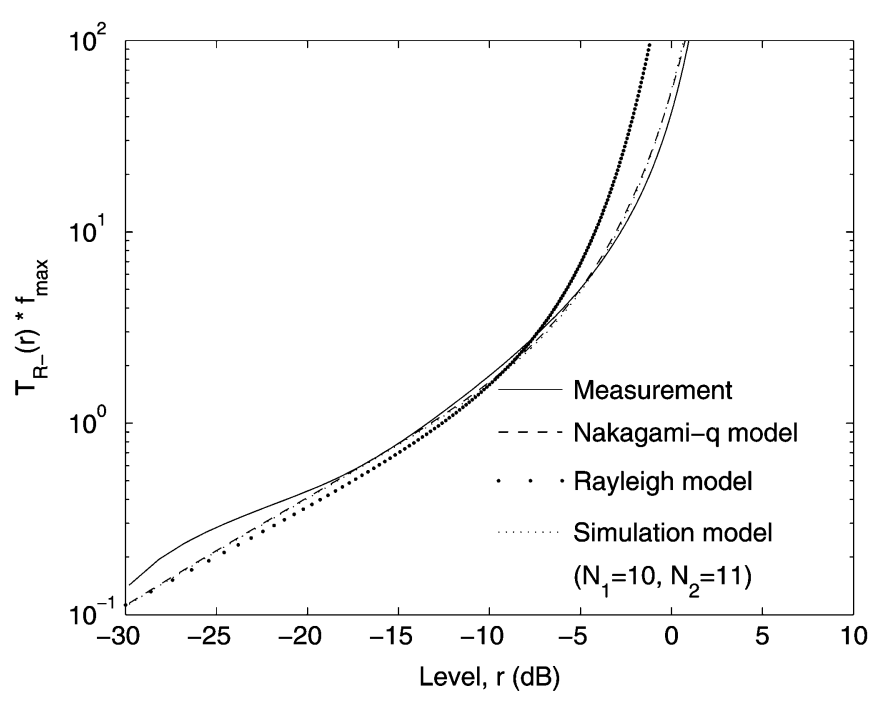

Fig. 6. Normalized average duration of fades $T_{R-}(r) \cdot f_{\max }$ for heavy shadowing.

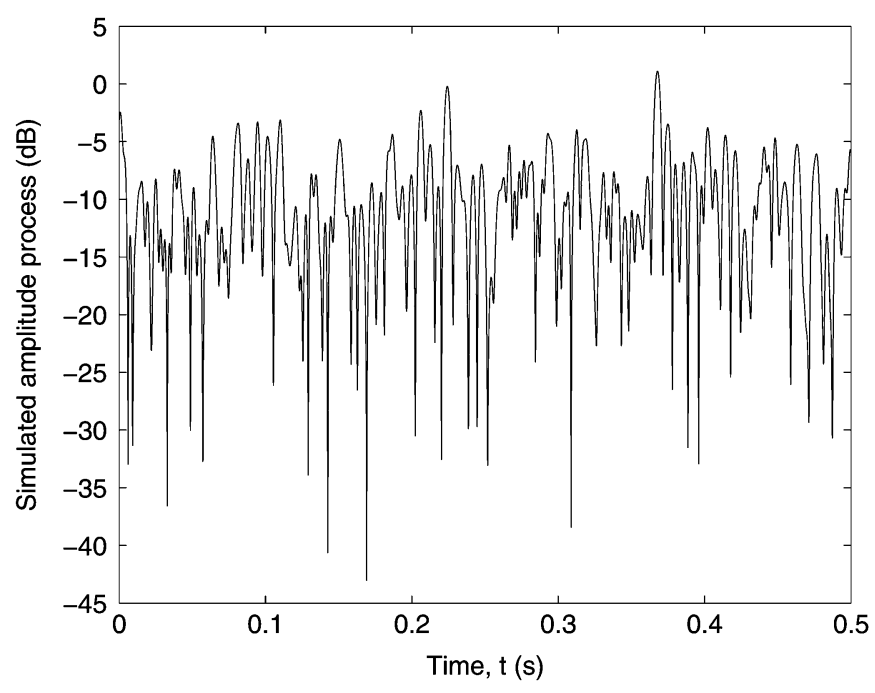

Fig. 7. A waveform example of the simulated deterministic process $\tilde{R}(t)$.

results reported in [28] for the heavy shadowing case, it is noted that the difference between the measured LCR, and the corresponding results obtained from both the analytical model and the simulation model is large. As for the results reported in [3], [29], and [30], it was shown therein that the models proposed can be used for the description of the statistics of the equivalent mobile satellite channel for an environment with heavy shadowing. The strength of the Nakagami- $q$ model, with respect to these models, lies in the simplicity of its simulation. Namely, the Nakagami- $q$ model simulator is just a slight modification of the Rayleigh fading channel simulator. This advantage is of great practical importance for the simulation and performance analysis of related mobile communication systems, such as the computation of the bit error probability.

Finally, a waveform example of the simulated envelope process, $\tilde{R}(t)=\left|\tilde{\mu}_{1}(t)+j \tilde{\mu}_{2}(t)\right|$, using the parameters given in
Table I, is plotted in Fig. 7. The values of the number of sinusoids $N_{1}$ and $N_{2}$ have been selected to be 10 and 11, respectively.

\section{CONCLUSION}

In this paper, the second order statistics of the NakagamiHoyt (Nakagami- $q$ ) fading channel is considered. Closed-form expressions for the LCR and ADF have been derived. The fitting of the derived expressions to the corresponding measured quantities demonstrates the ability of the Nakagami- $q$ model to describe the statistical behavior of real world mobile fading channels. A deterministic simulation model for the considered channel has also been presented. The validity of the simulator has been demonstrated by comparing the simulated complementary CDF and the second order statistics (LCR and ADF) against the corresponding analytical and measurement results.

\section{REFERENCES}

[1] H. Hashemi, "The indoor radio propagation channel," Proc. IEEE, vol. 81, no. 7, pp. 943-968, Jul. 1993.

[2] M. K. Simon and M. S. Alouini, "A unified approach to the performance analysis of digital communication over generalized fading channels," Proc. IEEE, vol. 86, no. 5, pp. 1860-1877, Sep. 1998.

[3] A. Abdi, W. C. Lau, M. S. Alouini, and M. Kaveh, "A new simple model for land mobile satellite channels: First and second order statistics," IEEE Trans. Wireless Commun., vol. 2, no. 3, pp. 519-528, May 2003.

[4] M. Nakagami, "The m-distribution-A general formula of intensity distribution of rapid fading," in Statistical Methods in Radio Wave Propagation. New York: Pergamon, 1960, pp. 3-36.

[5] R. S. Hoyt, "Probability functions for the modulus and angle of the normal complex variate," Bell Syst. Tech. J., vol. 26, pp. 318-359, Apr. 1947.

[6] P. J. Crepeau, "Uncoded and coded performance of MFSK and DPSK in Nakagami fading channels," IEEE Trans. Commun., vol. 40, no. 3, pp. 487-493, Mar. 1992.

[7] A. Annamalai, C. Tellambura, and V. K. Bhargava, "Simple and accurate methods for the outage analysis in cellular mobile radio systems-A unified approach," IEEE Trans. Commun., vol. 49, no. 2, pp. 303-316, Feb. 2001.

[8] A. Mehrnia and H. Hashemi, "Mobile satellite propagation channel part II-A new model and its performance," in IEEE Veh. Technol. Conf. (VTC'99), Amsterdam, The Netherlands, 1999, pp. 2780-2784.

[9] N. Youssef, W. ElBahri, M. Pätzold, and S. ElAsmi, "On the crossing statistics of phase processes and random FM noise in Nakagami-q mobile fading channels," IEEE Trans. Wireless Commun., vol. 4, no. 1, Jan. 2005, pp. 24-29.

[10] S. O. Rice, "Distribution of the duration of fades in radio transmission," Bell Syst. Tech. J., vol. 37, pp. 581-635, 1958.

[11] W. C. Jakes, Microwave Mobile Communications, 2nd ed., Piscataway, NJ: IEEE Press, 1993.

[12] M. Pätzold, U. Killat, F. Laue, and Y. Li, "On the statistical properties of deterministic simulation models for mobile fading channels," IEEE Trans. Veh. Technol., vol. VT-47, no. 1, pp. 254-269, Feb. 1998.

[13] K. W. Yip and T. S. Ng, "A simulation model for Nakagami-m fading channels $m<1$," IEEE Trans. Commun., vol. 48, no. 2, pp. 214-221, Feb. 2000.

[14] Q. T. Zhang, "A decomposition technique for efficient generation of correlated Nakagami fading channels," IEEE J. Sel. Areas Commun., vol. 18, no. 11 , pp. 2385-2392, Nov. 2000.

[15] Z. Song, K. Zhang, and Y. L. Guan, "Generating correlated Nakagami fading signals with arbitrary correlation and fading parameters," in Proc. IEEE ICC'02, New York, NY, 2002, pp. 1363-1367.

[16] L. Jianxia and J. R. Zeidler, "A statistical simulation model for correlated Nakagami fading channels," in Proc. WCC-ICCT'00, Beijing, China, Aug. 2000, pp. 1680-1684.

[17] C. D. Iskander and T. P. Mathiopoulos, "Analytical level crossing rates and average fade durations for diversity techniques in Nakagami fading channels," IEEE Trans. Commun., vol. 50, no. 8, pp. 1301-1309, Aug. 2002. 
[18] K. Ohtani and H. Omori, "Distribution of burst error lengths in Rayleigh fading radio channels," Electron. Lett., vol. 16, no. 23, pp. 889-891, 1980.

[19] K. Ohtani, K. Daikoku, and H. Omori, "Burst error performance encountered in digital land mobile radio channel," IEEE Trans. Veh. Technol., vol. VT-23, no. 1, pp. 156-160, 1981.

[20] H. S. Wang and N. Moayeri, "Finite state Markov channel-A useful model for radio communication channels," IEEE Trans. Veh. Technol., vol. VT-44, no. 1, pp. 163-171, Feb. 1995.

[21] C. Tepedelenlioglu, A. Abdi, G. B. Giannakis, and M. Kaveh, "Estimation of Doppler spread and signal stength in mobile communications with applications to handoff and adaptive transmission," Wirel. Commun. Mobile Comput., pp. 221-242, 2001.

[22] A. Abdi and M. Kaveh, "Level crossing rate in terms of the characteristic function: A new approach for calculating the fading rate in diversity systems," IEEE Trans. Commun., vol. 50, pp. 1397-1400, Sep. 2002.

[23] N. Youssef, T. Munakata, and M. Takeda, "Fade statistics in Nakagami fading environments," in ISSSTA'96, Mainz, Germany, Sep. 1996, pp. 1244-1247.

[24] J. S. Butterworth and E. E. Matt, "The characterization of propagation effects for land mobile satellite services," in Inter. Conf. Satellite Systems for Mobile Communication and Navigations, Jun. 1983, pp. 51-54.

[25] R. Fletcher and M. J. D. Powell, "A rapidly convergent descent method of minimization," Comput. J., vol. CJ-6, no. 2, pp. 163-168, 1963.

[26] D. F. Shanno, "Conditioning of quasi-Newton methods for function minimization," Math. Comput., vol. 24, pp. 647-656, 1970.

[27] W. H. Press, B. P. Flannery, S. A. Teukolsky, and W. T. Vetterling, Numerical Recipes in C, The Art of Scientific Computing. Cambridge, U.K: Cambridge Univ. Press, 1990.
[28] C. Loo, "A statistical model for land mobile satellite link," IEEE Trans. Veh. Technol., vol. VT-34, no. 3, pp. 122-127, Feb. 1985.

[29] M. Pätzold, U. Killat, and F. Laue, "An extended Suzuki model for land mobile satellite channels and its statistical properties," IEEE Trans. Veh. Technol., vol. VT-47, no. 2, pp. 617-630, May 1998.

[30] M. Pätzold, U. Killat, Y. Li, and F. Laue, "Modelling, analysis, and simulation of nonfrequency selective mobile radio channels with asymmetrical Doppler power spectral density shapes," IEEE Trans. Veh. Technol., vol. VT-46, no. 2, pp. 494-507, May 1997.

Neji Youssef, photograph and biography not available at the time of publication.

Cheng-Xiang Wang, photograph and biography not available at the time of publication.

Matthias Pätzold, photograph and biography not available at the time of publication. 